\title{
The UCAC as Input Catalog for FAME
}

\author{
N. Zacharias ${ }^{1}$, T.J. Rafferty, S.E.Urban, M.I. Zacharias ${ }^{2}$ and \\ G.L. Wycoff \\ U.S. Naval Observatory (AD), Washington, DC 20392
}

\begin{abstract}
A new, highly accurate, astrometric star catalog is available from the U.S. Naval Observatory, covering $\approx 80 \%$ of the Southern Hemisphere to $\mathrm{R} \approx 16^{m}$. The observing program is ongoing and full-sky coverage is expected by 2003 . The final catalog will be used as the input catalog for the FAME space mission.
\end{abstract}

\section{Introduction}

The USNO CCD Astrograph Catalog (UCAC) project is a high precision, astrometric survey of the 7-16 magnitude range (579-642 $\mathrm{nm}$ bandpass), aiming at an accuracy of 20 mas for $9^{m}-14^{m}$ stars. UCAC data have an average density (stars per square degree) higher than that of the Guide Star Catalog (GSC), with an improvement in positional accuracy of about a factor of 10 . UCAC data are about a factor of 5 more precise than the USNO A2.0 catalog (Monet et $a l ., 1999)$, however about a factor of 10 less dense.

\section{Observations}

The observations are made with a $0.2 \mathrm{~m}, 5$-element astrograph equipped with a $4 \mathrm{k} \times 4 \mathrm{k}$ CCD. The project started in January 1998 at the Cerro Tololo InterAmerican Observatory (CTIO), and observations are continuing. The instrument will be moved north in early 2001, and full-sky coverage is expected by mid-2003. More details about the telescope and the camera are listed in Table 1.

Figure 1 shows the survey fields which are completed as of April, 2000. In addition to the regular survey fields, about 30 calibration fields are being observed very often. A dedicated effort to link the extragalactic radio reference frame to the optical system is part of the program, using the CTIO $0.9 \mathrm{~m}$ and LNA $1.6 \mathrm{~m}$ telescopes.

\footnotetext{
${ }^{1}$ nz@pisces.usno.navy.mil

${ }^{2}$ also with Universities Space Research Association (USRA), Washington, DC
} 


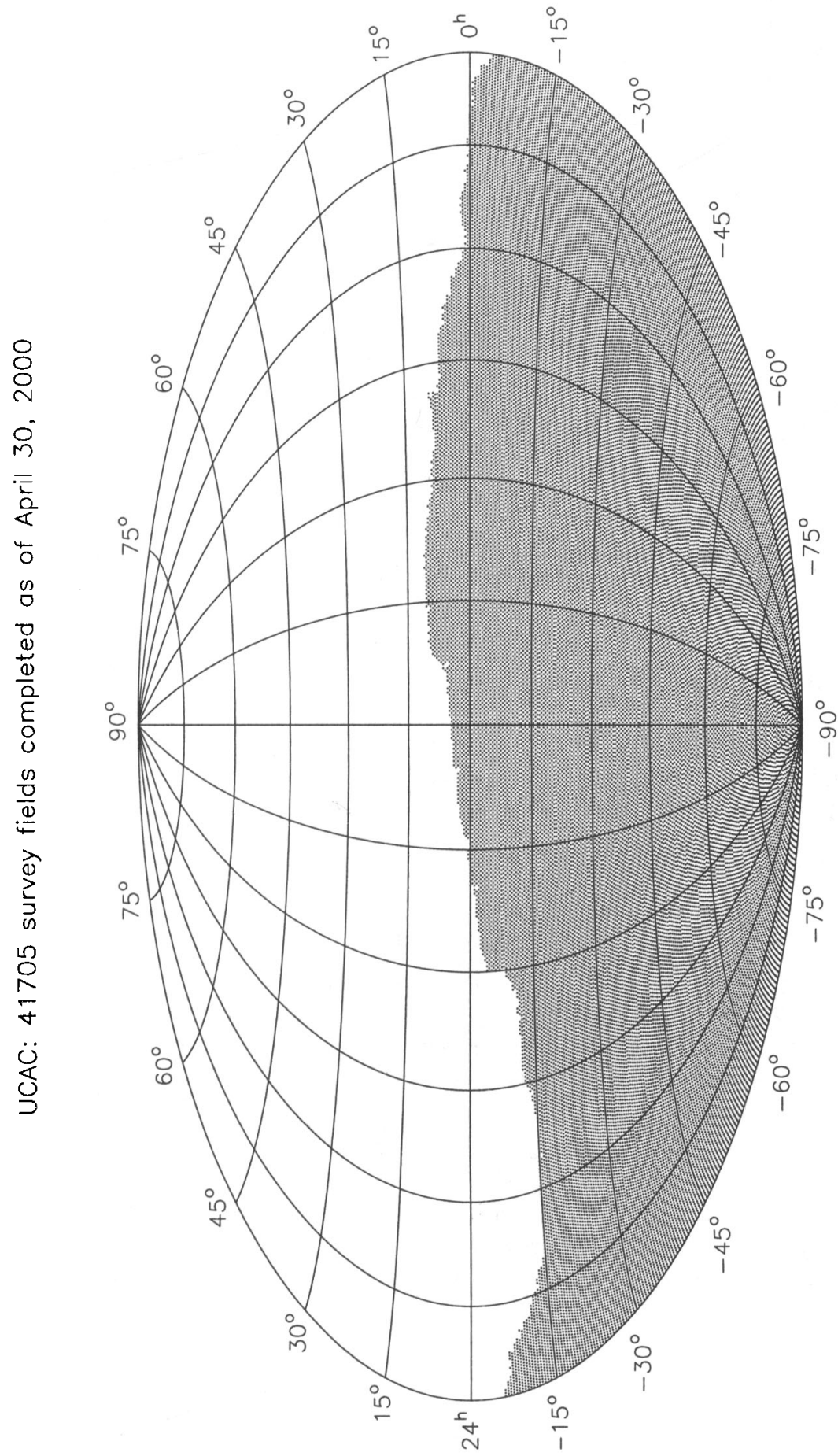

Figure 1. Completed survey fields on an equal-area Aitoff projection in equatorial coordinates. 
Table 1. Telescope and Camera.

\begin{tabular}{lrl}
\hline clear aperture & 206 & $\mathrm{~mm}$ \\
focal length & 2057 & $\mathrm{~mm}$ \\
number of lens elements & 5 & \\
spectral bandpass of lens & $550-710$ & $\mathrm{~nm}$ \\
Airy disc diameter (610nm) & 15 & $\mu \mathrm{m}$ \\
usable flat field of view & $\approx 9$ & degree \\
\hline number of pixels & $4094 \times 4094$ & \\
field of view & $61 \times 61$ & arcmin \\
pixel scale & 0.905 & $" /$ pixel \\
spectral bandpass used & $579-642$ & $\mathrm{~nm}$ \\
readout & 14 & $\mathrm{bit}$ \\
readout noise & 13 & $e^{-}$ \\
full well capacity & 85,000 & $e^{-}$ \\
operating temperature & $\approx-18$ & $\mathrm{C}$ \\
\hline
\end{tabular}

\section{The first catalog}

A preliminary catalog (UCAC1) of positions and proper motions of 27 million stars has been constructed, which is available on CD-ROM from USNO. Details about the project and the this first catalog can be found in Zacharias et al. (2000a). Observations from 13 Feb 1998 through 07 Nov 1999 are included with a total of over $79,000 \mathrm{CCD}$ frames. The catalog is on the International Celestial Reference System (ICRS), which is consistent with J2000. This first catalog is not complete, e.g. all problem stars have been omitted. The standard errors in position of UCAC1 are shown in Figure 2.

Proper motions of bright stars $(V \leq 12.5)$ were derived using a combination of ground-based astrometric catalogs, Hipparcos, and Tycho-2 positions, giving a typical error of $3 \mathrm{mas} / \mathrm{yr}$. For the fainter stars the USNO A2.0 (Monet et al., 1998) was used as first epoch, with typical proper motion errors of 10 and 15 mas/yr. Thus the distribution of UCAC1 proper motion errors is bi-modal, as shown in Figure 3.

Systematic errors in the UCAC positions are small (10 mas level), as external comparisons with SPM 2.0 (Platais et al., 1998) and the Tycho-2 Catalogue (Høg et al., 2000) revealed. Details are found in Zacharias et al. (2000b).

\section{FAME}

The Full-Sky Astrometric Mapping Explorer (FAME) to be launched in 2004 requires an input catalog for stars to 15 th magnitude with a positional standard error of $\approx 100$ mas or below. The final UCAC catalog is expected to be available at that time. The limiting magnitude of the UCAC $\left(R \approx 16^{m}\right)$ will be sufficient. Currently, UCAC is the only project which can meet all requirements for the FAME input catalog, providing full-sky coverage will be realized in time. 


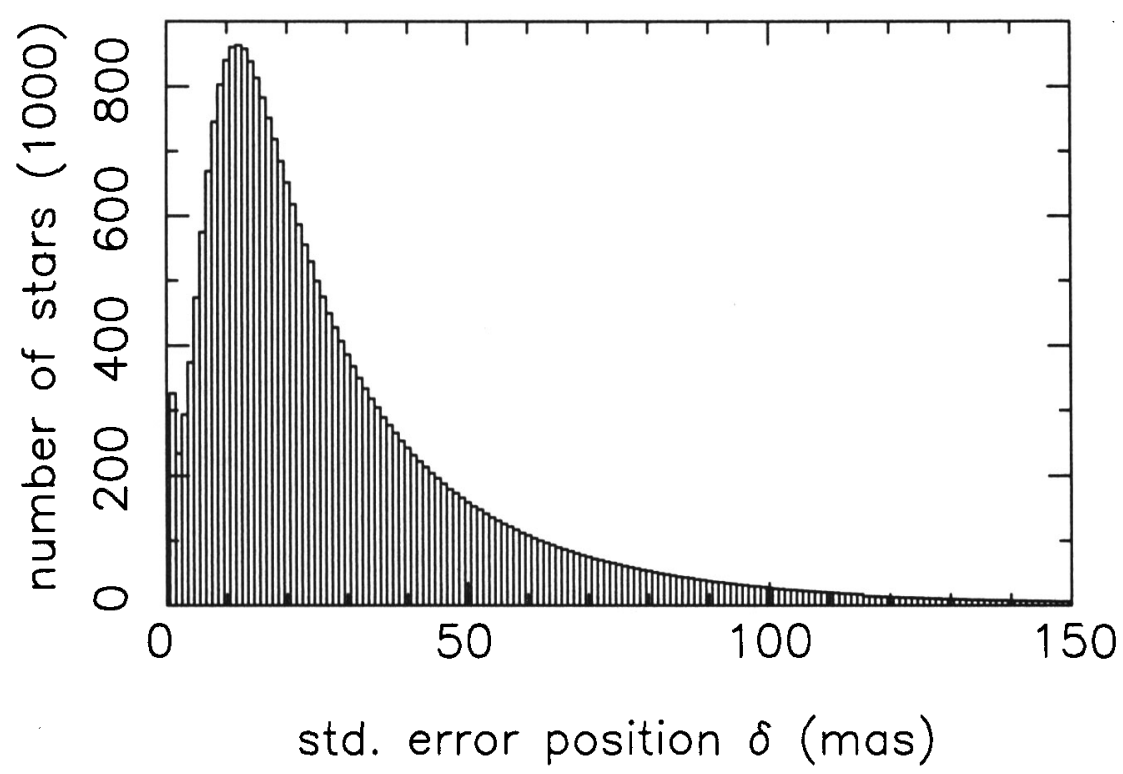

Figure 2. Distribution of the formal positional errors (declination) of all UCAC1 stars.

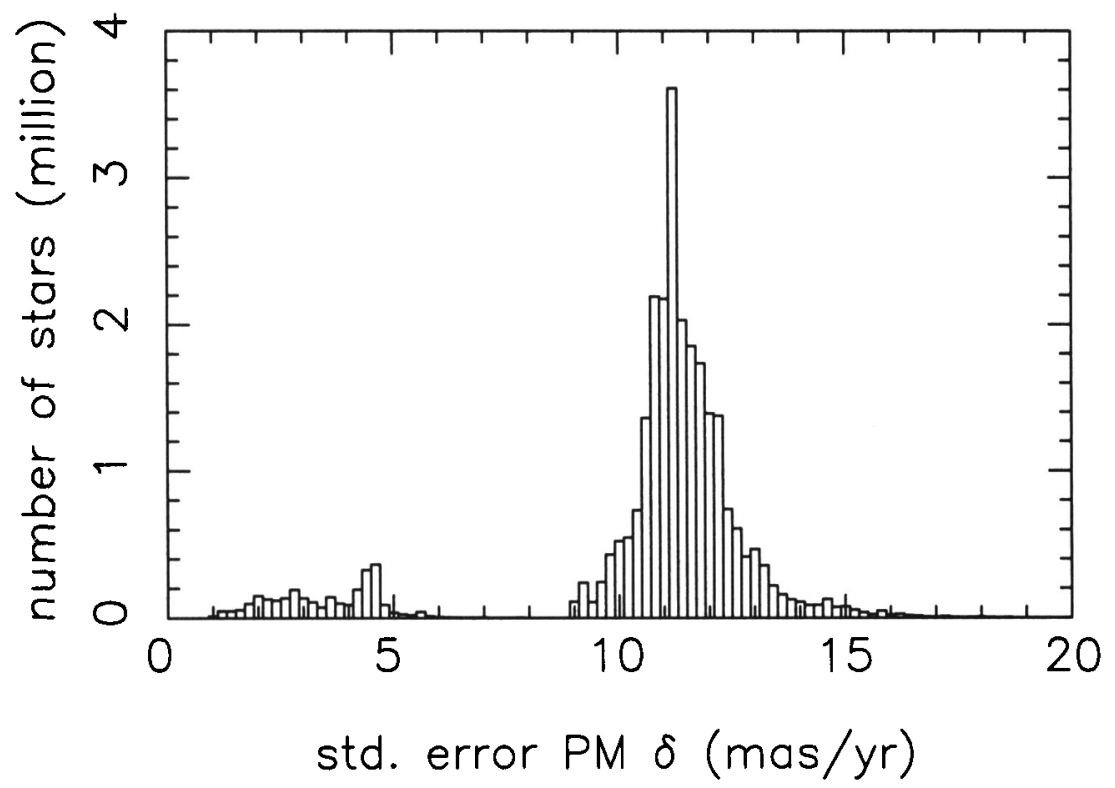

Figure 3. Distribution of the proper motion errors (declination) of all UCAC1 stars. 


\section{Outlook}

Significant updates in the software are in progress to further reduce systematic errors in the derived positions, handle galaxies and nebulae, crowded fields and to resolve most wide double stars. A particular problem is the unique match of images from various catalogs. A given epoch difference and confusion limit can only detect a certain range of proper motions. The UCAC observing program is designed to achieve a high level of completeness for stellar sources fainter than 8th magnitude. Improved proper motions on the 4 mas/yr level for all stars will be obtained by a new reduction of all applicable Northern and Southern Proper Motion survey (NPM \& SPM; Lick, Yale / San Juan) plates. Scanning of all those plates on PMM (USNO, Flagstaff) will be complete later this year.

Acknowledgments. We are grateful to the entire UCAC team and the CTIO staff, in particular the observers D. Castillo and M. Martinez. CTIO is operated by NOAO/AURA and supported by the National Science Foundation (NSF).

\section{References}

Høg, E., Fabricius, C., Makarov, V.V., Bastian, U., Schwekendiek, P., Wicenec A., Urban, S., Corbin T., \& Wycoff, G., 2000, Astron. Astrophys., in press.

Johnston, K.J.(PI) 1999, Full-sky Astrometric Mapping Explorer, FAME Concept Study Report, US Naval Observatory, Washington DC.

Monet, D.G. et al., 1999, USNO A2.0 catalog on CD-ROM.

Platais et al. 1998, Astron. J., 116, 2556, see also, http://www.astro.yale.edu/spm/spm2cat/spm2.html.

Zacharias, N., Urban, S.E., Zacharias, M.I., Hall, D.M., Rafferty, T.J., Germain, M.E., Holdenried, E.R., Pohlman, J.W., Gauss, F.S., Monet, D.G. \& Winter, L., 2000a, submitted to Astron. J.

Zacharias, N., Zacharias, M.I., Urban, S.E. \& Høg, E., 2000b, Astron. J. in press. 